\title{
MANEJO DE RECURSOS CULTURALES Y PUESTA EN VALOR DE HISTORIAS REGIONALES.*
}

\author{
Mónica Berón** \\ Marina Guastavino***
}

\begin{abstract}
Resumen: Desde el año 2002 se esta desarrollando un Plan de Manejo de Recursos Culturales (MCR) en el marco de un convenio interinstitucional en el sur de la provincia de La Pampa (República Argentina). El mismo se lleva a cabo en la localidad de Puelches y tiene como objetivos principales el uso racional, rentable y sustentable de los recursos culturales (APN 2000). El patrimonio, conformado por estos recursos, es la base fundamental de la identidad de un pueblo. En este mismo sentido se orientan los resultados de la investigación básica del pasado prehispánico del área. Recientemente un proyecto turístico impulsado por el gobierno central de La Pampa ha dado un giro a distintos aspectos del proyecto, que proponemos discutir en este trabajo.
\end{abstract}

Palabras clave: Manejo de Recursos Culturales; museo de la localidad; museo de sitio; Villa Casa de Piedra; Grandes Obras.

\section{Introducción}

Desde el año 2002 se está desarrollando un Plan de Manejo de Recursos Culturales en el marco de un convenio interinstitucional en el sur de la provincia de La Pampa (República Argentina). El mismo tiene como centro de actividades la localidad de

\footnotetext{
* Trabajo presentado en la IV Reunión Internacional de Teoría Arqueológica en América del Sur, Inter-Congreso del WAC (World Archaeological Congress - Congreso Arqueológico Mundial), Catamarca, Argentina, julio 2007. ** Conicet, Museo Etnográfico, UBA, Incuapa, UNCPBA. monberon@mail.retina.ar. Moreno 350, (1091) Ciudad de Buenos Aires, Argentina

*** Museo Etnográfico, UBA.

marinaguastavino@yahoo.com
}

Puelches y como objetivos principales el uso racional, rentable y sustentable de los sitios culturales (APN 2000, Figura 1). Se ha asumido la existencia de una interrelación dinámica entre patrimonio, identidad y conservación (Molinari et al. 2000). El patrimonio, conformado por estos recursos, es la base fundamental de la identidad de un pueblo. Una condición necesaria para cualquier estrategia orientada al uso sustentable de la biodiversidad y a la revalorización de los recursos naturales y culturales sería en este entendimiento, la definición y reconstrucción de su identidad. En este mismo sentido se orientan los resultados de la investigación básica del pasado prehispánico del área, que sin embargo es una zona marginal para los intereses del gobierno provincial. 


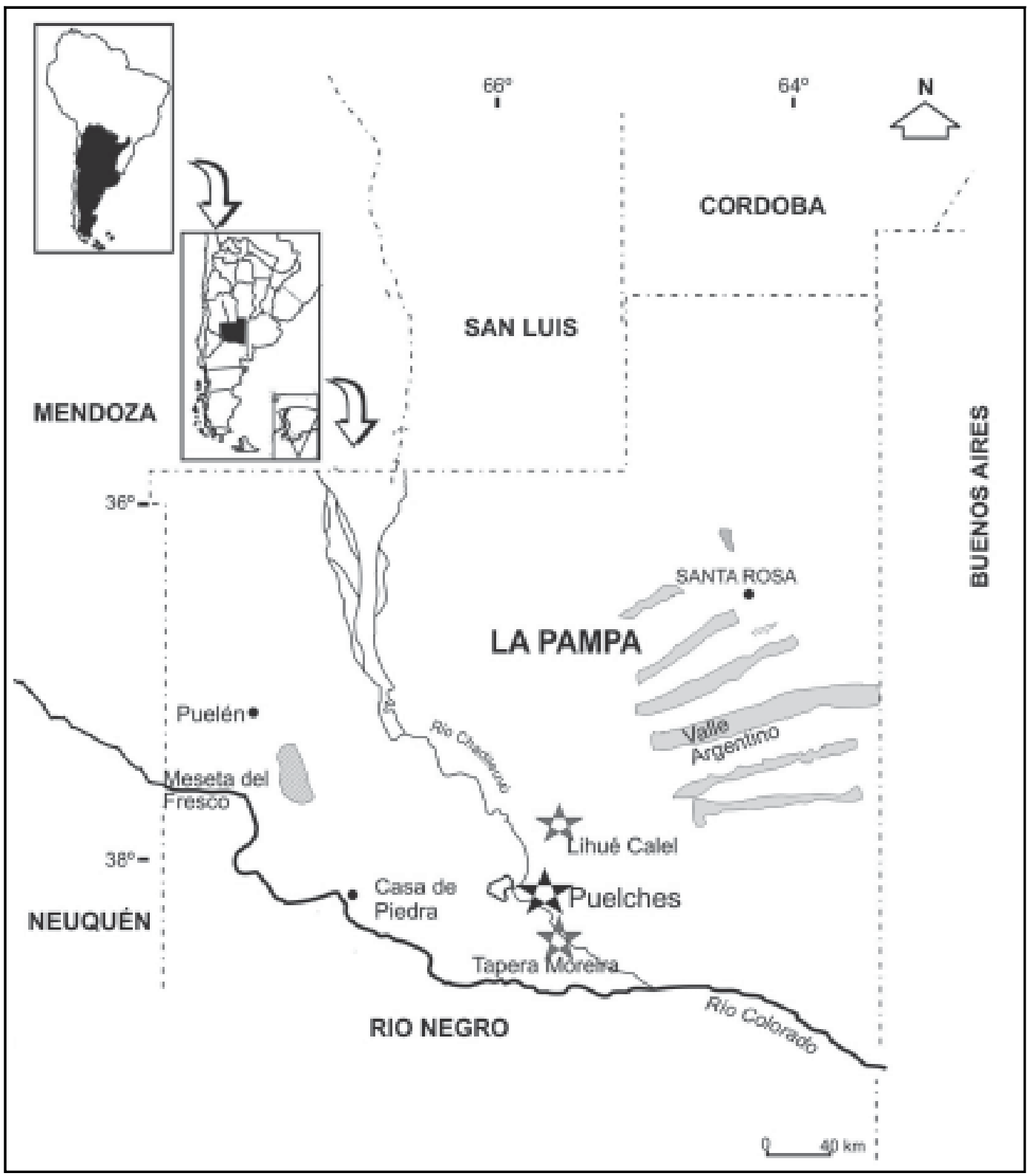

Fig. 1 - Ubicación de Puelches y de algunos puntos de localización de patrimonio arqueológico del área.

Recientemente un proyecto turístico del gobierno central de La Pampa ha puesto en el centro de las discusiones algunos de los objetivos del plan de manejo referido, lo que ha dado un giro a distintos aspectos del proyecto, que proponemos discutir en el marco de este simposio. El presente trabajo tiene como objetivo problematizar las acciones implementadas en el marco del Proyecto "Gestión de Patrimonio Cultural y Natural en la Comunidad de Puelches, Provincia de La Pampa: Conservación y Desarrollo". 


\section{La localidad de Puelches. Realidades y aspiraciones}

La localidad de Puelches dista $280 \mathrm{kms}$ de Santa Rosa, la capital provincial. Está ubicada en pleno semidesierto pampeano, área también conocida como "la travesía" en los registros históricos. Tiene 400 habitantes distribuidos entre el ejido urbano y el área rural. Su emplazamiento responde a la disponibilidad de fuentes de agua superficial, como las cuencas de los ríos Curacó y Chadileuvú y el sistema lagunas constituido por Urrelauquen, La Dulce y La Amarga. Sin embargo, aunque en distinta medida, todos estos cuerpos de agua presentan altos niveles de salinidad, por lo cual no son potables. La localidad recibe el agua potable a través de un acueducto que se extiende desde Puelén hasta Puelches y recorre más de 150 $\mathrm{km}$. Hasta hace pocos años dicha localidad resultaba un punto aislado en la travesía hacia el Alto Valle de Río Negro, ya que la ruta $N^{\circ} 152$ se hallaba en malas condiciones y ninguna línea de transporte de pasajeros pasaba por allí sino solamente por la Ruta del Desierto.

A partir de mejoras en la Ruta Nro. 152 se produjo un tránsito mayor de aquellos que viajan desde y hacia la Patagonia y pasan por la localidad de Puelches, que se ubica a ambos lados de dicha ruta. Se consideró que estas mejoras permitirían el desarrollo de la comunidad. Se instaló una estación de servicio y algunos paradores, a instancias de emprendimientos de pobladores locales. Paralelamente algunos pobladores han planificado la instalación de un balneario en la laguna La Dulce, con un lugar de recreación, que aún no logra concretarse.

\section{EI MRC en el marco de proyectos de desarrollo regional}

El Manejo de Recursos Culturales (MRC) es una estrategia que se pone en práctica para el cuidado del Patrimonio Cultural. Este patrimonio es el que engloba el conjunto de los recursos culturales, que entendemos en los términos de R. Molinari (1998) como aquellas evidencias materiales de las actividades de los hombres a lo largo de la historia, en la interacción entre ellos y con el medio que los rodea. Asimismo, dichos componentes del patrimonio son las evidencias por las cuales una comunidad se reconoce a sí misma y es reconocida por los demás (Molinari 1998).

Entendemos, además, que cuidar el patrimonio implica realizar una serie de acciones que se encuentran interrelacionadas, como investigación, conservación y puesta en valor. Ahora bien, la conservación de los recursos culturales de una comunidad no implica una preservación absoluta, sin la participación de los involucrados, sino el "uso racional, rentable y sustentable", tendiente al desarrollo y al mejoramiento de la calidad de vida de dicha comunidad.

De esta forma, poner en valor un recurso cultural es conservarlo en la acepción antes descripta. Lo mencionado anteriormente debe llevarse a cabo en el marco de un trabajo participativo. Por eso, se hace necesario plantear espacios en los que el intercambio horizontal de conocimientos y opiniones, faciliten la toma de decisión de los distintos actores de una comunidad.

Si bien existe en la provincia de La Pampa, una intención de fortalecer la identidad de la zona del oeste y/o del desierto, que se refleja en el cancionero, en la poética, en la literatura y en algunas acciones políticas puntuales, no existe una política integral tendiente al desarrollo socioproductivo que, entre otras cosas, contribuya a disminuir el éxodo rural.

Recientemente, se lanzó el Proyecto de la Villa Casa de Piedra que, aparentemente, contribuiría al desarrollo mencionado en el párrafo anterior.

\section{Proyecto Villa Casa de Piedra}

En el año 2004 el gobierno de la Pcia. de La Pampa presentó el Plan Director del Ente 
Comunal Casa de Piedra. La creación de esta nueva comuna, oficiada por Ley № 2112 del 08/07/2004, tiene como eje la creación de un "Polo productivo - turístico con toda la infraestructura y los servicios necesarios para el desarrollo de un lugar estratégico en la Patagonia Argentina". La nueva villa se ubica a $396 \mathrm{~km}$ al suroeste de la capital pampeana; sobre las márgenes del Río Colorado, a orillas de un lago artificial de 36.000 hectáreas de superficie. A la misma se accede por Ruta Nacional № 152 o Ruta Provincial № 34. Es un ambiente de semidesierto, de tipo patagónico, con bardas y terrazas fluviales con escasa vegetación xerófila (Figura 2).

El proyecto se está desarrollando en etapas; la primera ya está en marcha y consiste en el establecimiento de un Área Prioritaria Urbana que brinda la infraestructura necesaria: grupo de viviendas, caminos, energía, comunicación, alumbrado, agua potable, riego, forestación, parquización y bajada para lanchas.

En la Figura 3 pueden observarse los cambios en la fisonomía del ambiente a partir de la implementación del riego para forestar. La intencionalidad en este caso no es mostrar y conservar el ambiente natural sino recrear un paisaje artificial.

En la segunda etapa, el sector privado comenzará a realizar sus inversiones en los servicios planificados según el proyecto: hoteles, canchas y zonas deportivas, un club náutico, venta de productos y comidas regionales, estación de servicio, parador de camiones y micros, restaurante, área comercial, camping, etc. La inversión pública incluirá además un balneario, miradores, centro de salud, destacamento policial, escuela

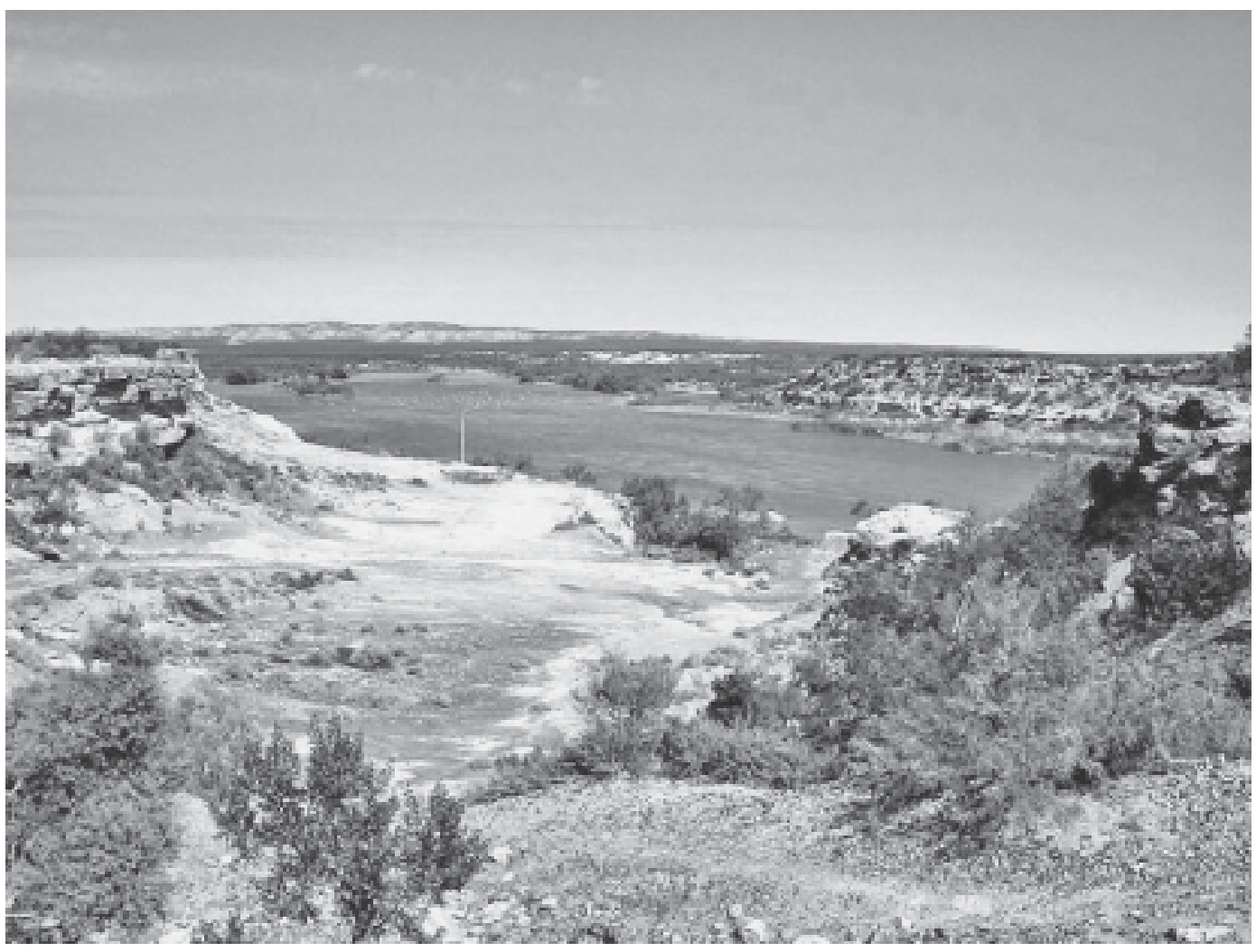

Fig. 2 - Bardas y terrazas sobre el Río Colorado. 


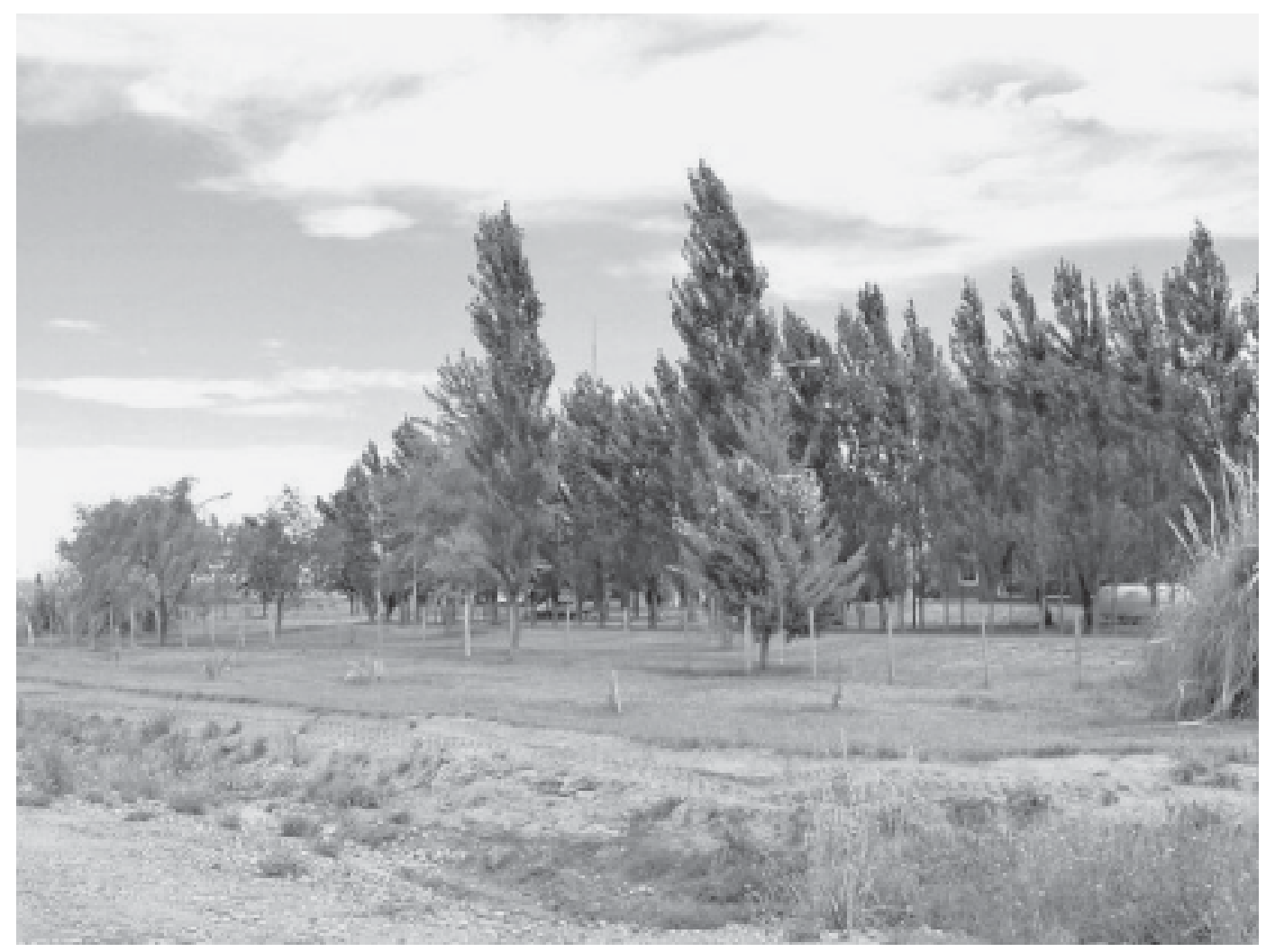

Fig. 3 - Parquización en el entorno de la Villa.

y viviendas. Además, se proyecta la construcción del "Museo del Hombre Pampeano" en el cual se exhibirán las colecciones recuperadas en el área durante las tareas de evaluación de impacto arqueológico realizadas entre 1978 y 1986, en el marco del proyecto Presa Embalse Casa de Piedra, por Gradín y colaboradores. Su premisa es "contribuir con la construcción de la identidad pampeana a través de la difusión de los primeros momentos de su historia, considerando que forma parte de los múltiples procesos, hitos y actores que confluyen y se suceden en el devenir histórico para conformar la realidad en que vivimos" (Becerra, M. L. Pera y M. I. Poduje 2004).

El Ente Comunal Casa de Piedra está conformado por seis zonas donde se desarrollan distintos usos (Figura 4).
Cabe destacar que la Secretaría de Turismo de la Provincia de La Pampa, presenta el proyecto de la siguiente manera:

\section{"VI LLA TURÍ STI CA “CASA DE PI EDRA”}

\section{DESPUES DE 76 AÑOS UN NUEVO PUEBLO NACE EN LA PAMPA...}

El verde de la esperanza y el agua de la vida se instalaron definitivamente en Casa de Piedra; el paisaje es una invitación a quedarse y pertenecer al lugar."

Mientras desde la esfera oficial del gobierno provincial se alienta a todos los sectores a formar parte de un exitoso proyecto en el cual "habrá trabajo para to- 


\section{Z O N I F I C A C I O N}

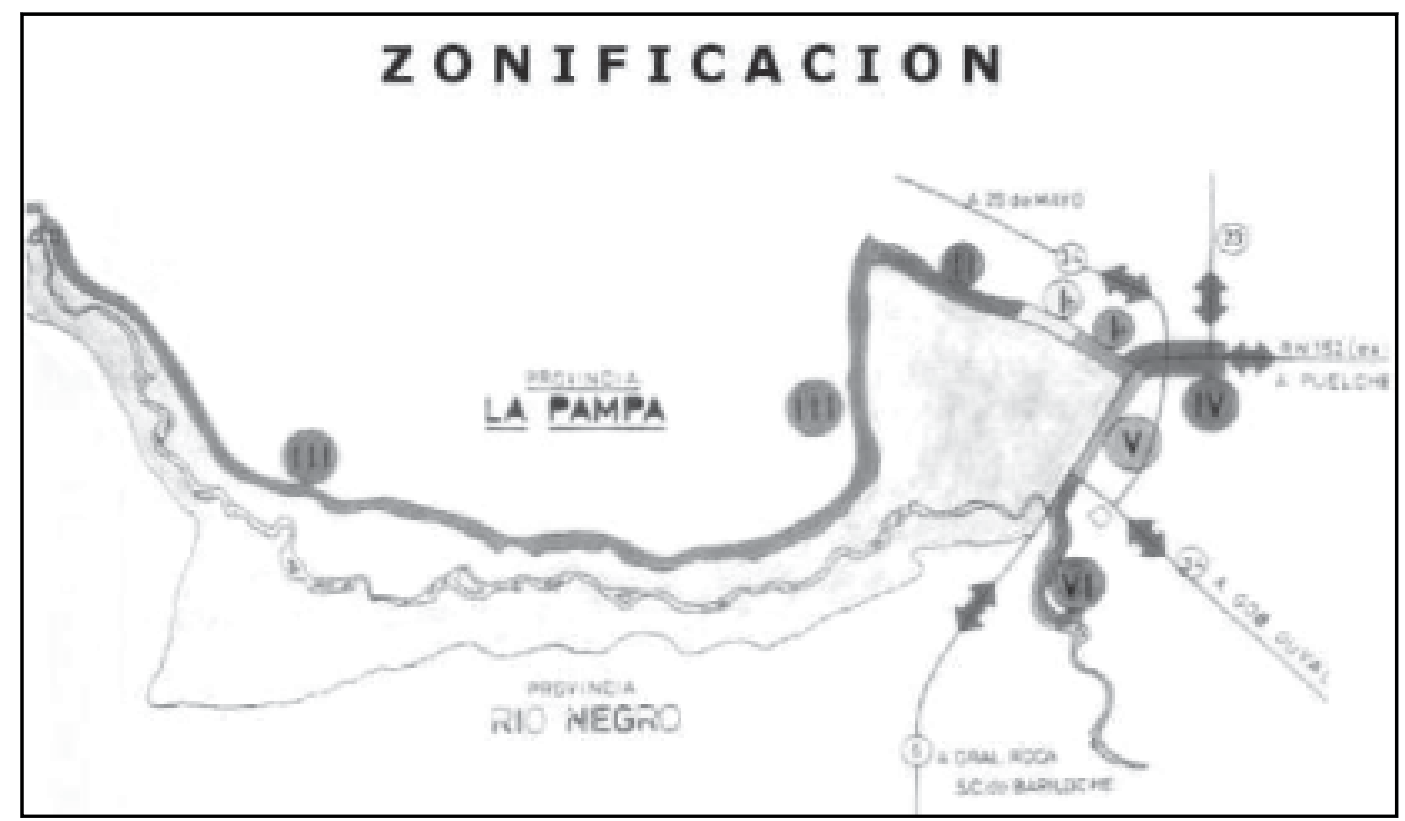

Fig. 4 - Villa Casa de Piedra. Referencias:

- Sector 1a -1b: Desarrollo de un Área Prioritaria Urbana para el Desarrollo del Turismo, Deporte y Recreación. Proyecto de Riego y Forestación.

- Sector II: Desarrollo de un Área de Producción Agrícola-Ganadera.

- Sector III: Reserva: Área Protegida.

- Sector IV: Portal de Acceso a Casa de Piedra y Puesto de Control de Fauna y Flora.

- Sector V: Aguas Abajo de la Presa. Forestación paisajista y riego.

- Sector VI: Costa del Río Colorado: Mirador; Estación de Piscicultura.

dos (sic)", tal como fue presentado en la 320 Feria del Libro en Buenos Aires (pampavirtual $15 / 3 / 2006$ ), en medios nacionales como el diario La Nación se referenció al proyecto como "Las Vegas del subdesarrollo" (La Nación, 1/12/2006). Entre los proyectos figura la instalación de un casino y un hotel 5 estrellas, que seguramente no están destinados a los pobladores locales, ni pensados para el desarrollo del Oeste pampeano, que registra una densidad poblacional menor que la de los mayores desiertos del mundo y en constante retroceso.

\section{Totalidad vs. Fragmentación}

Consideramos que un proyecto que tiene como finalidad mejorar la calidad de vida de las poblaciones, debería partir de considerar la realidad como totalidad. Encontramos que las nociones que priman son las de fragmentación, donde la realidad es segmentada en fragmentos, considerando la suma de ellos como la totalidad. La consideración de forma separada de esos fragmentos, tanto por la ciencia como por la política, la economía, etc., ha llevado a obtener una serie de resultados negativos. Es cierto que dividir temas y trabajos ha permitido tratar con la realidad, reduciendo problemas a proporciones manejables. No obstante ello, debemos tender hacia "modos de contemplar el mundo como un todo (..) más que como "conocimiento absolutamente verdadero de cómo son las cosas" (Bohm 1998:24-25). Es decir, debemos ser conscientes que lo que es real es la totalidad y que la fragmentación 
es la estrategia que el hombre despliega, dada su tendencia a pensar el mundo en forma fragmentaria.

Asimismo, consideramos la realidad conformada por totalidades menores que se van combinando entre sí y que a su vez son incluidas en y forman parte de una totalidad más grande. Al emprender acciones sobre una de esas totalidades menores, no solemos tener en cuenta que estamos trabajando sobre una más grande y que esas acciones van a afectarla subsecuentemente. (Savory y Butterfield, 1999). Entonces, deberíamos empezar tratando de entender esa totalidad mayor que posee características que no podemos observar en las totalidades menores que la conforman. Esta perspectiva holística, permitiría acercarnos más eficientemente sobre las totalidades menores sobre las que se ha decidido implementar determinadas acciones. Partiendo de la noción de que el todo es la realidad, "el todo debe ser definido, teniendo en mente que siempre ha influenciado y fue influenciado por las totalidades mayores y menores, y que tuvimos que conocer qué queríamos hacer con él: que necesitábamos una toda-abarcadora meta holística". (Savory y Butterfield, 1999:9).

\section{La gestión del patrimonio cultural - es- tado actual}

En el año 2002 se firmó un convenio interinstitucional para llevar adelante un proyecto en la localidad de Puelches, en la provincia de La Pampa, República Argentina. El Proyecto "Gestión de Patrimonio Cultural y Natural en la Comunidad de Puelches, Pcia. de La Pampa: Conservación y Desarrollo" responde a un compromiso entre la Administración de Parques Nacionales (APN) - Consejo Nacional de Investigaciones Científicas y Técnicas (CONICET) - Programa de Investigaciones Arqueológicas del Cuaternario Pampeano (INCUAPA) - Asociación ALI HUEN - Instituto Nacional de Tecnología Agropecuaria (INTA)-Subsecretaria de Cultura de la Provincia de La Pampa-Municipalidad de
Puelches-Subsecretaría de Turismo- Instituto de Estudios Sociohistóricos de la Facultad de Ciencias Humanas, Universidad Nacional de La Pampa (UNLPam.).

El objetivo del presente proyecto consiste en términos generales, en establecer el manejo participativo de los recursos culturales y naturales de la localidad de Puelches, atendiendo al desarrollo socio-económico de la zona. En ese marco se concretaron diversos emprendimientos que surgieron a partir de las necesidades planteadas por los participantes de los talleres de evaluación, que se realizan en la localidad, aproximadamente tres veces al año. Hasta el momento se han realizado 11 talleres (Figura 5).

Desde los inicios en el año 2002, el proyecto fue sufriendo diferentes ajustes como así también la incorporación de nuevas instituciones a su desarrollo. Paralelamente, se produjeron cambios en la coyuntura político-social a nivel provincial y local que han incidido en mayor o menor medida en el desarrollo de las acciones propias del proyecto.

Los avances obtenidos hasta el momento, consisten en:

1. Traslado y acondicionamiento del taller de artesanas e inicio de comercialización de la producción (Figuras 6 y 7).

2. Impresión y distribución de folletos turísticos

3. Inicio de la Actividad de la Historia Oral, con el fin de fortalecer y recuperar la identidad a través del desarrollo de actividades que permitan interrelacionar la memoria individual con la historia colectiva recuperando y actualizando los conocimientos sobre el medio cultural, natural y social.

4. Relevamiento de documentación para la edición de un libro de historia de la comunidad (a cargo del Instituto de Estudios Sociohistóricos, Facultad de Ciencias Humanas, UNLPam.) 


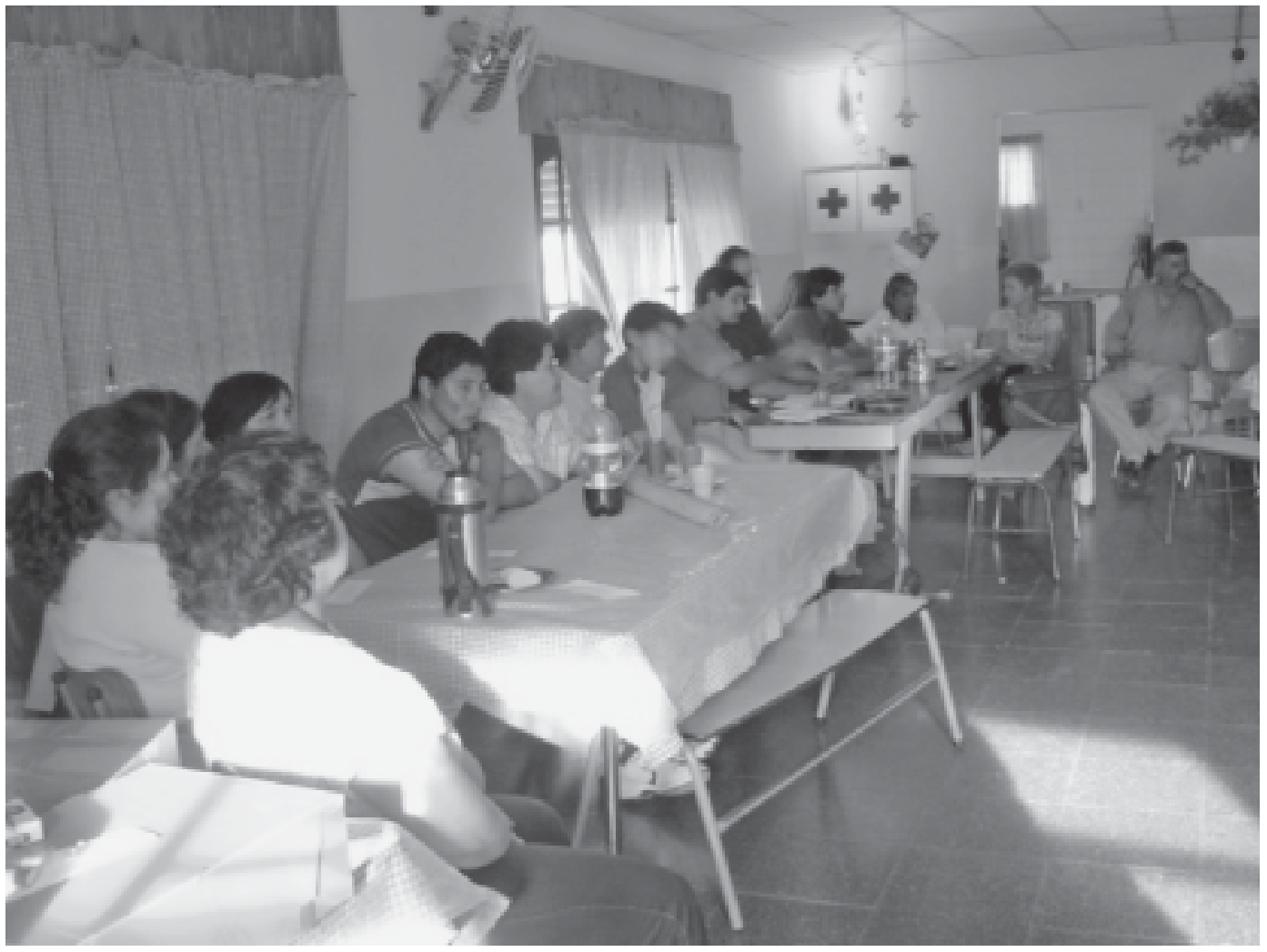

Fig. 5 - Talleres de Evaluación

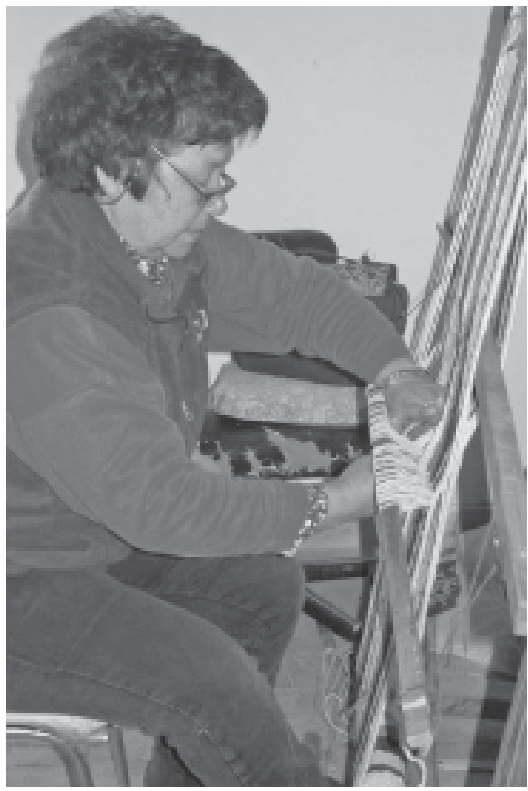

5. Acondicionamiento de la antigua capilla para instalación de un museo de la localidad:

Desde los primeros contactos con los pobladores en el marco de los trabajos de investigación arqueológica se percibió en algunos miembros de la comunidad su deseo de rescatar la historia local a través de la instalación de un Museo de la Localidad.

De esta manera ha sido una de las propuestas del vigente proyecto de investigación: estudiar, reconstruir y revitalizar la historia del proceso de colonización de fines del Siglo XIX y poblamiento del Siglo XX de las áreas involucradas en este proyecto, rescatando el conjunto de tradiciones y actividades

Fig. 6 - Artesana tejiendo en telar vertical. 


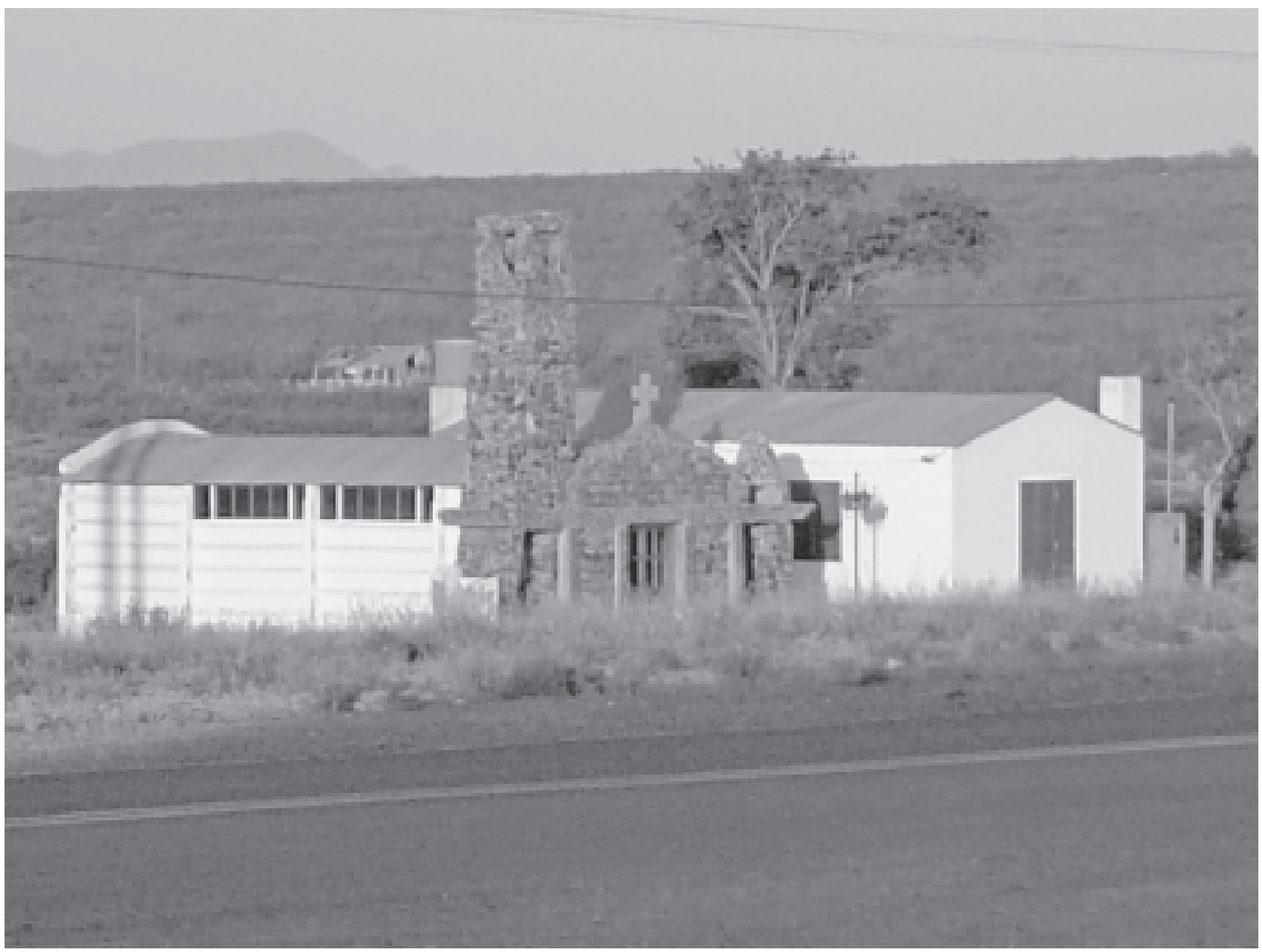

Fig. 7 - A la izquierda, local del Museo de la localidad, a la derecha, taller de artesanos.

económicas y sociales que caracterizó a esa época, desde los primeros colonizadores. Se pretende rescatar el conocimiento de los antiguos pobladores del área rural y/o de sus descendientes, así como el sistema de ideas y valores recreado en torno a los sitios prehispánicos. Entre los objetivos se incluye comparar la información arqueológica del proceso de colonización con los datos contenidos en los documentos históricos, crónicas y registros de la época y rescatar la historia regional a partir de entrevistas a las viejas familias y pobladores de la zona. Con este propósito se ha incorporado al equipo de investigación un estudiante avanzado de Antropología Social y por otra parte se ha conformado un grupo de investigación de la historia local a instancia del Instituto de Estudios Sociohistóricos de Facultad de Ciencias
Humanas (UNLPam), que convocaron dos pasantes a cargo de los cuales se halla el relevamiento de documentación para la recuperación de la historia regional. Paralelamente se ha avanzado en la redacción del guión del Museo y se ha comprado equipamiento para su montaje (PC multimedia, vitrinas), a través de un subsidio de la Secretaría de Cultura de la Nación. También se ha lanzado un concurso para la elección del nombre del Museo, entre los pobladores locales.

\section{Plan de manejo del Parque Nacional Lihué Calel, Provincia de La Pampa, Argentina}

Desde el año 1998 se están realizando investigaciones arqueológicas sistemáticas, que han arrojado información de gran 
relevancia acerca de los grupos cazadoresrecolectores que habitaron dicha región (Berón et al 2002, 2006).

El principal sitio arqueológico investigado se denomina Chenque I, un cementerio indígena prehispánico de cazadores-recolectores (Figura 8). Se ubica sobre una lomada baja, en un lugar destacado del paisaje, circundado por cerros altos y lomadas menores. Hasta el momento, se presenta como uno de los cementerios más grandes de cazadoresrecolectores de pampa-patagonia. El sitio presenta un rango temporal de utilización que va del $1050 \mathrm{AP}$ al 320 años A.P.

Tiene una superficie de $210 \mathrm{~m}^{2}$, demarcada por rocas que conforman una estructura subcircular. Se ha excavado un total de $49 \mathrm{~m}^{2}$, lo que constituye el $23 \%$ del total. El número mínimo de individuos recuperados se ha calculado en 158 individuos, por lo cual se estima que varios cientos de

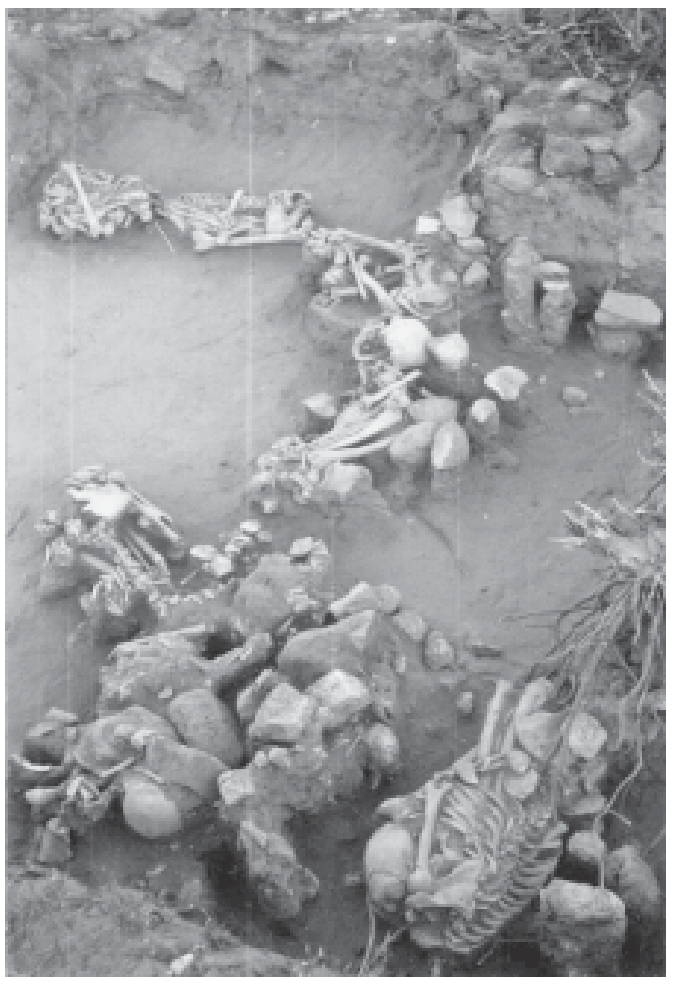

Fig. 8 - Enterratorios del sitio Chenque I. personas fueron enterradas allí a lo largo de 700 años de uso.

Otra perspectiva contemplada en el proyecto de MRC es la puesta en valor del sitio Chenque I y la transferencia de resultados, por medio del montaje de un Museo de Sitio. A través del mismo, se pretende dar a conocer al público la dimensión biológica del pasado cultural de la región, a partir de la recreación de las costumbres funerarias y de la caracterización bioarqueológica de los individuos enterrados en este cementerio. Dos premisas guiarán los contenidos de la muestra: recrear y respetar la sacralidad del sitio y poner en un plano de relevancia los modos de vida de los antiguos pobladores del desierto pampeano.

En la 'puesta en valor' interpretativa de los recursos culturales de Lihué Calel se apuntará a crear un acercamiento interactivo y comprensible del pasado, utilizando la combinación de aspectos naturales y culturales del ambiente (paisaje) en el espacio del museo de sitio seleccionado. Metodológicamente se ha planteado sostener un manejo adaptativo que fuera capaz de minimizar los riesgos de deterioro, poder anticipar consecuencias previstas y no previstas que sucedan a la aplicación de intervenciones sobre los recursos culturales, y avanzar en el manejo, incorporando a su re-planificación la información de los resultados parciales (Molinari 1998).

\section{Consideraciones finales}

Los efectos de las Grandes Obras tienen un impacto no sólo local sino regional y además se prolongan en el tiempo (Radovich 2003). Un proyecto de desarrollo de gran magnitud como el mencionado en Casa de Piedra, debería plantearse indicadores de impacto que permitan evaluar las consecuencias (tanto negativas como positivas) que resulten de esas acciones. En este sentido, creemos necesaria la definición de indicadores de impacto para las localidades vecinas a la Villa Casa de Piedra y, en este caso particular, 
para la localidad de Puelches. Como antecedente se debe mencionar la evaluación de impacto arqueológico y antropológico llevado a cabo en los inicios del proyecto de construcción de la Presa Embalse, a solicitud del Ente Ejecutivo tripartito Casa de Piedra. La evaluación de impacto arqueológico se desarrollo entre 1978 y 1986 y estuvo a cargo del Prof. Carlos J. Gradin y un amplio grupo de colaboradores, entre ellos una de las autoras (MB) (Gradin et al 1984). El impacto antropológico se desarrolló entre 1986 y 1988 y estuvo a cargo de un grupo de geógrafos encabezados por la Dra. N. Medus (Medus et al. 1988).

Entre los postulados del actual proyecto se incluye "impulsar un nuevo polo turístico y productivo en un lugar de óptimas distancias con importantes capitales del país y sitio estratégico para el paso a los destinos vacacionales del resto de la Patagonia (Ruta Nacional № 152). Además se integra al circuito turístico del cercano Parque Nacional Lihué Calel y, más lejana, la Reserva Provincial Parque Luro."

Esta frase sintetiza el marco de las expectativas que este nuevo mega- proyecto regional ha generado en los pobladores y autoridades locales, particularmente en Puelches. Esas expectativas no van de la mano del acompañamiento de nuestro proyecto de MRC, que lo precede en el tiempo pero que no logra la concreción de las pequeñas etapas y mini-inversiones que la Intendencia local se compromete a efectuar respecto al Museo de la localidad y Taller de Artesanos. (rejas de seguridad, alarma, personal a cargo). Sin embargo se impulsó vivamente la construcción de un polideportivo de grandes dimensiones, junto a la ruta 152 , para cuya inauguración estuvo presente el gobernador de la provincia. También se ha iniciado la construcción de un tambo caprino, con un crédito de promoción productiva destinado al propietario de las instalaciones. En palabras del Intendente de Puelches, con motivo de la inauguración: "Hoy estamos inaugurando el primer tambo experimental demostrativo de secano en la provincia de La Pampa (...) Podemos decir que un producto autóctono del oeste pampeano como es la producción caprina, ha cumplido el sueño de pasar a ser una producción sustentable y generadora de mano de obra, permitirá que más productores caprineros puedan agregarle valor a su producción ${ }^{2 "}$.

En este punto se considera necesario establecer indicadores que midan el impacto de cada uno de estos emprendimientos, en escala multidimensional, sobre la calidad de vida de los pobladores (económicos, sociales y ambientales), a fin de lograr esa visión de la realidad total e integradora en la que el patrimonio cultural se constituya como un recurso de desarrollo.

Como dice Endere (2001), dar participación a la población local en la administración del patrimonio constituye un desafío pero también una tarea que parece depender cada vez más del voluntariado y la sensibilidad de la sociedad civil, y nos obliga a los arqueólogos a asumir un papel más activo, mediando entre los distintos grupos de interés. En medio de todo este panorama, ¿cómo se inserta la mirada hacia el pasado? Mientras un sector clama por la recuperación de la memoria y el rescate de los ancestros, otros sólo están pendientes de la gran obra de inversión hacia un futuro que no está asegurado. 


\begin{abstract}
Cultural Research Management Program (CRM) is being developed since 2002 in the south of La Pampa province (Argentina) framed in an inter-institutional agreement. This program is being developed in Puelches locality and the first objectives are the rational, income- producing and maintainable use of cultural resources (APN 2000). The heritage, composed by these resourcesis the base of a population identity. In the same way are considered the results of prehispanic past research carried out in the area. Recently, a tourist project impelled by the central governmet of La Pampa province has redirected different aspects of our CRM program, which we discuss in this paper.
\end{abstract}

Key words: Cultural Research management, locality museum, site museum, Casa de Piedra village, Big Dams.

\title{
Bibliografia
}

BECERRA, M., L. PERA Y M. I. PODUJE

2004 Proyecto Museo Provincia "Carlos J. Gradin". Departamento Investigaciones Culturales, Subsecretaría de Cultura, Ministerio de Cultura y Educación, Gobierno de La Pampa,

BERÓN, M., I. BAFFI, R. MOLINARI, C. ARANDA, L. LUNA, A. CIMINO

2002 El chenque de Lihue Calel. Una estructura funeraria en las Sierras de la Vida. En Del Mar a los Salitrales. 10.000 de Historia Pampeana en el Umbral del Tercer Milenio: 87-106. Mazzanti, D., M. Berón y F. Oliva Eds. Universidad Nacional de Mar del Plata. Facultad de Humanidades. Laboratorio de Arqueología. Mar del Plata. Código de ISBN: 987- 544- 052- 3.

BERÓN M., L. LUNA Y R. CURTONI

2006 Serranías Pampeanas Meridionales. Investigaciones arqueológicas en el área de Lihué Calel. En Incuapa, 10 años. G. Politis Ed. FACSO Olavarría

BOHM, D.

1998 La totalidad y el orden implicado. Cap.1: Fragmentación y totalidad. 3a. Edición Kairós. Barcelona

ENDERE, M.L.

2001 Patrimonio arqueológico en Argentina. Pa- norama actual y perspectivas futuras. Revista de Arqueología Americana20: 143-158.

GRADÍN, C., C. VAYÁ, M. QUINTANA, H. NAMI, A.

SALVINO, M. BERÓN Y A. AGUERRE

1984 Investigaciones Arqueológicas en Casa de Piedra. Pcia. de La Pampa. Dirección General de Cultura y Ente Ejecutivo Casa de Piedra.

MEDUS, N. L. MORETE Y G. TOURN

1988 Presa Embalse Casa de Piedra. Relocalización de Población. Informe Final. Santa Rosa, La Pampa; M/S:

MOLINARI, R.; L. FERRARO; H. A. PARADELA Y A. CASTAÑO.

2001 Odisea del Manejo: Conservación del Patrimonio Arqueológico y Perspectiva Holística. 2do. Congreso Virtual de Antropología y Arqueología. www.naya.org.ar

RADOVICH J.C

2003 Impacto social de grandes aprovechamientos hidroenergéticos sobre comunidades rurales de Norpatagonia. Tesis Doctoral no publicada. Facultad de Filosofía y Letras. UBA.

SAVORY, A. Y J. BUTTERFIELD

1999 Holistic Management: a New Framework for Decision Making. Island Press.2nd Edition. Washington 